\title{
Supply Chain Coordination for Fixed Lifetime Products with Permissible Delay in Payments
}

\author{
Yongrui Duan, Guiping Li, and Jiazhen Huo \\ School of Economics and Management, Tongji University, Shanghai 200092, China \\ Correspondence should be addressed to Yongrui Duan; yrduan@163.com
}

Received 12 February 2014; Revised 13 April 2014; Accepted 13 April 2014; Published 7 May 2014

Academic Editor: Ming Li

Copyright ( 2014 Yongrui Duan et al. This is an open access article distributed under the Creative Commons Attribution License, which permits unrestricted use, distribution, and reproduction in any medium, provided the original work is properly cited.

\begin{abstract}
This paper considers a two-level supply chain coordination problem for fixed lifetime products with permissible delay in payments. Two cases are discussed; that is, the retailer is required to settle the balance before the end of the ordering cycle (Case I) and after the ordering cycle (Case II). The coordination models are proposed and analyzed, respectively. The analytical methods as to how to determine the optimal policy are presented. In addition, it is indicated that the supplier's cost as well as that of the total system will be reduced no matter how much the parameters change, and the retailer will benefit from coordination, if the supplier is willing to share the cost saving with him/her in Case I. In Case II, the retailer's average cost will be reduced and the supplier will benefit from coordination only under certain conditions. Besides, the results show that, for fixed lifetime products, providing longer credit period than the retailer's order period is not commonly applicable.
\end{abstract}

\section{Introduction}

In recent years, the area of supply chain management has become very popular. Many researchers have devoted considerable attention to coordination issues between suppliers and retailers in a supply chain. A variety of coordination mechanisms are developed and utilized in the different settings.

Monahan [1] studied a single supplier-single buyer distribution system and indicated that the supplier could improve channel coordination and earn higher profits by offering the buyer a quantity discount. Later, Lee and Rosenblatt [2] generalized Monahan's model by relaxing his lot-for-lot assumption for the supplier. Shin and Benton [3] studied the effectiveness of quantity discounts under different conditions. They showed that the link between quantity discounts and the performance of the chain is influenced by several other factors including the variability of demand, the relative inventory cost structures, and the buyer's economic reorder intervals. Jaber and Osman [4] proposed a centralized model where the players in a two-level supply chain coordinated their orders through delay in payments to minimize their local costs and that of the chain. Chen and Kang [5] developed the integrated models with permissible delay in payment for determining the coefficient of negotiation and the maximum delay payment period. Sarmah et al. [6] studied a coordination mechanism through credit option such that both parties can divide the surplus equitably after satisfying their own profit targets. Emmons and Gilbert [7] studied the effect of return policies on both manufacturer and retailer's profits. In their model, demand is uncertain and price-dependent. Giannoccaro and Pontrandolfo [8] coordinated a three-stage supply chain with a stochastic customer demand by using the revenue sharing policy. Extensive reviews of the literature on supply chain coordination are available in $[9,10]$.

In the supply chain coordination literatures that have been mentioned above, the researchers all ignored the constraint of items' finite lifetime in their models. In fact, many products or goods have their own sell-by dates. Foodstuff, such as bread, milk, and beverage, all become unusable after a certain time. Photographic material, blood, and drugs are further examples of items with fixed lifetime. Moreover, the upgrade of IT products, the launch of new products, and the change of popularity make more and more products have a certain lifetime. In the past few decades, the inventory optimization of the finite lifetime product has been the focus of many researchers. Nahmias [11] classified the finite lifetime problem into two subcategories: random finite lifetime and fixed finite lifetime. The random finite lifetime products, such 
as fruit and vegetable, spoil over time and the value also decreases gradually. Items with the fixed finite lifetime, on the other hand, perish at the same age; that is, the value remains the same during the lifetime period and equals to zero after the expiration date. The relevant researches such as [12-17] are about the random finite lifetime products. Goyal and Giri [18] provided an excellent and detailed review of research on inventory problem of this kind of product. The researches about the fixed finite lifetime products can refer to [19-26] and their related references.

However, the above researches on fixed finite lifetime product are on single stage problem. Duan et al. [27] considered a single-vendor, single-buyer supply chain for fixed lifetime product and proposed models to analyze the benefit of coordinating supply chain through a quantity discount strategy. In addition, they proved that the quantity discount strategy can achieve system optimization and winwin outcome.

Delay in payment is one of the commonly used mechanisms in supply chain coordination practice. In real world, the supplier often utilizes this policy to promote his/her products, especially when the retailer is short of capital. In this policy, the supplier requires the retailer to increase his order size by offering the retailer a delay period. And the retailer is required to settle the balance before the end of the delay period. During the delay period, the retailer can cumulate revenues and earn interests. The policy can reduce the retailer's capital cost tied up in stock, because it reduces the amount of capital invested in stock for the duration of the delay period, so that the retailer's holding cost of finance is reduced. The policy can also decrease supplier's ordering cost, but it adds an additional dimension of default risk to the supplier.

Duan et al. [28] dealt with a supply chain coordination problem through delay in payments for items with fixed lifetime, where the costs of the supplier and retailer include the ordering cost and the storage cost. The opportunity cost of capital cost is not considered. However, when the products are kept in the warehouse, a large amount of capital will be backlogged, which will incur great opportunity cost in addition to the storage cost. Furthermore, in this paper, we consider supply chain coordination issues for fixed lifetime product through delay in payment. In the delay in payment contract, the supplier will provide a credit period for the retailer. In this case, the capital cost of the retailer is a nonlinear and a piecewise function of time. So it is necessary to investigate how the optimal policy is changed when capital cost is considered. The main differences between Duan et al. [28] and this paper are as follows. Firstly, in addition to the ordering and storage cost, this paper considers the opportunity cost of capital in the model formulation, which is not considered in [28]; secondly, because the retailer's opportunity costs of capital backlogging are dependent on the time when the balance are settled, we discuss two cases. Case I: the retailer is required to settle the balance before the end of the ordering cycle and Case II: the retailer settles the balance after the ordering cycle. Thirdly, we find that when the opportunity cost is not considered, the supplier will always benefit no matter how much the parameters change, and the cost of the retailer will not change. However, when the opportunity cost is considered and the retailer settles the balance after the ordering cycle, then the cost of the supplier may increase but the cost of the retailer may decrease. So the opportunity costs have significant influence on the optimal coordination policy.

The rest of this paper is organized as follows. In Section 2, the supply chain coordination model to minimize the supplier's cost is formulated, and the analytically tractable solutions are derived. A numerical study is conducted in Section 3 to illustrate the performance of the proposed policy and examine the implications of the change in the value of parameters. The concluding remarks are presented in the last section.

\section{Model Formulation}

In this section, we formulate and analyze the decisionmaking models of a two-level supply chain for fixed lifetime products without and with coordination. In the absence of coordination, both of the parties make decisions to minimize their own costs. In the presence of coordination, the supplier is the decision-maker of the supply chain with the objective of minimizing his/her own cost, and the delay in payments is adopted as a coordination policy. In addition, we obtain the optimal policies for the different cases and find that the lifetime of the product is an important constraint in the optimal policies.

We adopt the same assumptions as in Duan et al. [28] which are also commonly used in the literatures, see Monahan [1] and Lee and Rosenblatt [2].

(i) Demand is exogenous and constant. The main reason of this assumption is to simplify the model formulation and to derive the closed-form solution to the model.

(ii) Replenishment is instantaneous and lead time is zero. Obviously, if the lead time is constant and not zero, the supplier and retailer just need to place an order a period exactly equal to the lead time in advance [2], and the same results can be obtained.

(iii) Shortages are not allowed. For fixed lifetime products, due to their limited lifetime, the customers would purchase only when they are to be used up. If stockout happens, most of them will buy the substitute products. In addition, because the demand is assumed to be constant in this paper, the shortage can be eliminated by increasing the ordering quantity.

(iv) All items ordered by the supplier arrive new and fresh; that is, their age equals zero [29]. In fact, if the items' age is $l$ when they arrive, we only need to replace the product lifetime $L$ by $L=L-l$ in the following analysis.

Notations used in this paper are as follows:

$i$ denotes the subscript identifying a specific level in a supply chain $(i=s, r$, where $s=$ supplier and $r=$ retailer); 
$D$ denotes the constant demand rate;

$A_{i}$ is the ordering cost of level $i$;

$h_{i}$ is the holding cost of finance per unit per unit time, representing the cost of capital at level $i$ excluding the storage cost;

$s_{i}$ is the storage cost per unit per unit time at level $i$ excluding the holding cost;

$c_{i}$ is the procurement cost per unit for level $i$;

$k_{i}$ is the return on investment for level $i$;

$Q_{0}$ is the retailer's EOQ, and $t_{0}$ is the order period of the retailer in the absence of any coordination;

$L$ denotes the lifetime of product and $L \geq t_{0}$;

$T C_{s}$ and $T C_{r}$ are the average costs of the supplier and the retailer in the absence of coordination;

$\overline{T C_{s}}$ and $\overline{T C_{r}}$ are the average costs of the supplier and the retailer in the presence of coordination.

\section{Decision Variables}

$\mu$ denotes the supplier's order multiple in the absence of any coordination;

$K$ denotes the retailer's order multiple in the presence of coordination and $K Q_{0}$ is the retailer's new order size;

$t(K)$ denotes the credit period offered to the retailer, if he orders $K Q_{0}$ every interval of $K t_{0}$ (interest free period);

$\lambda$ denotes the supplier's order multiple in the presence of coordination.

2.1. Model Formulation for System without Coordination. In this subsection, the decision-making model for fixed lifetime products without coordination is formulated. The retailer and the supplier make decisions by minimizing their own costs.

In the absence of any coordination, the retailer's order quantity is simply the EOQ; that is,

$$
Q_{0}=\sqrt{\frac{2 D A_{r}}{h_{r}+s_{r}}} .
$$

Let $T C_{r}$ denote the retailer's average cost per unit time and let $t_{0}$ denote the retailer's optimal order period; then

$$
\begin{aligned}
T C_{r} & =\sqrt{2 D A_{r}\left(h_{r}+s_{r}\right)}, \\
t_{0} & =\sqrt{\frac{2 A_{r}}{D\left(h_{r}+s_{r}\right)}} .
\end{aligned}
$$

Since the retailer's order quantity is fixed at $Q_{0}$, the supplier is faced with a stream of demands, each with order size $Q_{0}$ and at fixed intervals of $t_{0}$. Given such a stream of demands, the supplier's economic order quantity should be some integer multiple of $Q_{0}$ [2]. Let $\mu Q_{0}$ denote the supplier's order quantity where $\mu$ is a positive integer.
The supplier's average inventory held up per unit time is

$$
\frac{\left[(\mu-1) Q_{0}+(\mu-2) Q_{0}+\cdots+Q_{0}+0 Q_{0}\right]}{\mu}=\frac{(\mu-1) Q_{0}}{2} .
$$

The supplier's cost per unit time is

$$
T C_{s}(\mu)=\frac{D A_{s}}{\mu Q_{0}}+\left(h_{s}+s_{s}\right) \frac{(\mu-1) Q_{0}}{2} .
$$

Hence, the supplier's problem in the absence of coordination can be formulated as follows:

$$
\begin{aligned}
& \min \quad T C_{s}(\mu)=\frac{D A_{s}}{\mu Q_{0}}+\left(h_{s}+s_{s}\right) \frac{(\mu-1) Q_{0}}{2} \\
& \text { s.t. } \quad \frac{\mu Q_{0}}{D} \leq L, \quad \mu \geq 1 \text { is an integer. }
\end{aligned}
$$

By substituting $Q_{0}$ into (5), we get

$$
\begin{aligned}
& \min T C_{s}(\mu)=\frac{A_{s}}{\mu} \sqrt{\frac{D\left(h_{r}+s_{r}\right)}{2 A_{r}}} \\
& +\left(h_{s}+s_{s}\right)(\mu-1) \sqrt{\frac{D A_{r}}{2\left(h_{r}+s_{r}\right)}} \\
& \text { s.t. } \quad \mu \sqrt{\frac{2 A_{r}}{D\left(h_{r}+s_{r}\right)}} \leq L, \quad \mu \geq 1 \text { is an integer. }
\end{aligned}
$$

The first constraint in (6) is to ensure that the products are not overdue before they are sold out. In this case, the supplier will minimize his average cost by selecting the optimal order multiple $\mu$. In the next section, we will give the analytical solution to (6).

Lemma 1. If $L \geq t_{0}=\sqrt{2 A_{r} / D\left(h_{r}+s_{r}\right)}$, then the supplier's optimal order multiple is as follows:

$$
\mu^{*}=\min \left\{\mu_{1}^{*}, \mu_{2}^{*}\right\}
$$

where $\mu_{1}^{*}=\left\lceil\sqrt{1 / 4+A_{s}\left(h_{r}+s_{r}\right) / A_{r}\left(h_{s}+s_{s}\right)}-1 / 2\right\rceil, \mu_{2}^{*}=$ $\left[L \sqrt{D\left(h_{r}+s_{r}\right) / 2 A_{r}}\right],\lceil x\rceil$ denotes the least integer greater than or equal to $x ;[x]$ denotes the integer part of $x$.

The proof of Lemma 1 can refer to Duan et al. [28]. Lemma 1 gives the supplier's optimal order multiple of the retailer's ordering quantity without coordination. Here, $L \geq$ $t_{0}$ is to ensure that the EOQ model is to ensure that the items are not expired before they are sold out.

2.2. Model Formulation for System with Coordination. In this subsection, we will propose and discuss the supply chain coordination model for fixed lifetime products through delay in payments policy. In this policy, the retailer orders $K Q_{0}$ units products every interval of $K t_{0}$ from the supplier at a unit purchasing $\operatorname{cost} c_{r}$ and the ordering cost $A_{r}$. The supplier orders $\lambda K Q_{0}$ units every interval of $\lambda K t_{0}$ and offers the credit 
period $t(K)$ to the retailer, where $K, \lambda$, and $t(K)$ are decision variables. Meanwhile, the retailer has the opportunity to invest the unpaid balance $c_{r} K Q_{0}$ for a period $t(K)$ at a return rate of $k_{r}$. That is, if the retailer can settle his balance at the time $t(K)$, they will have a capital gain of $c_{r} K Q_{0} k_{r} t(K)$, but the supplier's cost will be increased by $c_{r} K Q_{0} k_{s} t(K)$.

Therefore, the supplier's cost per unit time $\overline{T C_{s}}(K, \lambda)$ is composed of the following:

(i) the supplier's ordering cost per unit time which is equal to $D A_{s} / \lambda K Q_{0}$,

(ii) the supplier's holding cost of finance per unit time which is equal to $h_{s} K Q_{0}(\lambda-1) / 2+h_{s} D t(K)$,

(iii) the supplier's storage cost per unit time which is equal to $s_{s} K Q_{0}(\lambda-1) / 2$,

(iv) the supplier's opportunity cost per unit time which is equal to $c_{r} D k_{s} t(K)$.

Hence,

$$
\begin{aligned}
\overline{T C_{s}}(K, \lambda)= & \frac{D A_{s}}{\lambda K Q_{0}}+\frac{\left(h_{s}+s_{s}\right) K Q_{0}(\lambda-1)}{2} \\
& +h_{s} D t(K)+c_{r} D k_{s} t(K) .
\end{aligned}
$$

The retailer's cost consists of the following:

(i) the retailer's ordering cost per cycle which is equal to $A_{r}$,

(ii) the retailer's holding cost of finance per cycle which is equal to

$H_{r}(K)= \begin{cases}h_{r} \frac{\left(K Q_{0}-D t(K)\right)^{2}}{2 D}, & 0 \leq t(K) \leq K t_{0} \\ 0, & t(K) \geq K t_{0}\end{cases}$

(iii) the retailer's storage cost per cycle which is equal to $s_{r} K^{2} Q_{0} t_{0} / 2$,

(iv) the retailer's revenue during the credit period which is equal to $c_{r} K Q_{0} k_{r} t(K)$.

Therefore, the retailer's cost per unit time is

$$
\begin{aligned}
\overline{T C_{r}}(K) & =\frac{A_{r}+H_{r}(K)+s_{r} K^{2} Q_{0} t_{0} / 2-c_{r} K Q_{0} k_{r} t(K)}{K t_{0}} \\
& =\frac{D A_{r}}{K Q_{0}}+\frac{D H_{r}(K)}{K Q_{0}}+\frac{s_{r} K Q_{0}}{2}-c_{r} D k_{r} t(K) .
\end{aligned}
$$

To entice the retailer to accept this policy, the supplier should ensure that the retailer's average cost per unit time with coordination is not greater than that without any coordination. The coordination problem can be formulated as the following mathematical programming:

$$
\begin{array}{ll}
\min & \overline{T C_{s}}(K, \lambda) \\
\text { s.t. } & \lambda K t_{0} \leq L \\
& \overline{T C_{r}}(K) \leq T C_{r} \\
& \lambda \geq 1 \text { is an integer. }
\end{array}
$$

The first constraint of (11) is to guarantee that the supplier's products are not overdue before they are sold out. The second constraint is to ensure that the policy proposed by the supplier is acceptable to the retailer.

Based on the value of $H_{r}(K)$, two cases are discussed, respectively, in the following, namely, Case I: $0 \leq t(K) \leq K t_{0}$ and Case II: $t \geq K t_{0}$.

Case I. When $0 \leq t(K) \leq K t_{0}, H_{r}(K)=h_{r}\left(K Q_{0}-D t(K)\right)^{2} /$ $2 D$.

In this case, (11) is reduced to the following problem:

$$
\begin{array}{cl}
\min \quad & \overline{T C_{s}}(K, \lambda)=\frac{D A_{s}}{\lambda K Q_{0}}+\frac{\left(h_{s}+s_{s}\right) K Q_{0}(\lambda-1)}{2} \\
& +h_{s} D t(K)+c_{r} D k_{s} t(K) \\
\text { s.t. } \quad & \lambda K \frac{Q_{0}}{D} \leq L \\
& \frac{D A_{r}}{K Q_{0}}+\frac{h_{r}\left(K Q_{0}-D t(K)\right)^{2}}{2 K Q_{0}}+\frac{s_{r} K Q_{0}}{2} \\
& -c_{r} D k_{r} t(K) \leq \sqrt{2 D A_{r}\left(h_{r}+s_{r}\right)}
\end{array}
$$

$\lambda \geq 1$.

Theorem 2. Let $\mu^{*}$ and $\lambda^{*}$ be the supplier's optimal order multiple without and with coordination, and let $K^{*}$ be the retailer's optimal order multiple with coordination; then

$$
\overline{T C_{s}}\left(K^{*}, \lambda^{*}\right) \leq T C_{s}\left(\mu^{*}\right) \text {. }
$$

Proof. It is easy to verify that $\overline{T C_{s}}(K, \lambda)$ is increasing in $t(K)$. $\overline{T C_{r}}(K)$ is convex and decreasing in $t(K)$, for $0 \leq t(K) \leq K t_{0}$. As a result, the objective function of (12) is minimized when the second constraint is an equation; that is,

$$
\begin{gathered}
\frac{D A_{r}}{K Q_{0}}+\frac{D h_{r}}{K Q_{0}} \frac{\left(K Q_{0}-D t(K)\right)^{2}}{2 D}+\frac{s_{r} K Q_{0}}{2} \\
-c_{r} D k_{r} t(K)=\sqrt{2 D A_{r}\left(h_{r}+s_{r}\right)} .
\end{gathered}
$$

By rearranging the terms, we have

$$
\frac{h_{r}}{2}(D t(K))^{2}-\left(h_{r}+c_{r} k_{r}\right) K Q_{0} D t(K)+A_{r} D(K-1)^{2}=0 .
$$

Let $t_{1} \leq t_{2}$ be the two roots of (15); then, if $\left(h_{r}+c_{r} k_{r}\right)^{2} Q_{0}^{2} K^{2}-$ $2 h_{r} A_{r} D(K-1)^{2} \geq 0$,

$t_{1}=\frac{\left(h_{r}+c_{r} k_{r}\right) K Q_{0}-\sqrt{\left(h_{r}+c_{r} k_{r}\right)^{2} K^{2} Q_{0}^{2}-2 h_{r} A_{r} D(K-1)^{2}}}{h_{r} D}$,

$t_{2}=\frac{\left(h_{r}+c_{r} k_{r}\right) K Q_{0}+\sqrt{\left(h_{r}+c_{r} k_{r}\right)^{2} K^{2} Q_{0}^{2}-2 h_{r} A_{r} D(K-1)^{2}}}{h_{r} D}$. 
Since $\left(h_{r}+c_{r} k_{r}\right) K Q_{0}>0$ and $A_{r} D(K-1)^{2}>0$, for $K>1$, then $t_{1}>0$ and $t_{2}>0$. In addition, by noting that $t^{*}(K) \leq K Q_{0} / D$, we have

$t^{*}(K)$

$$
=\frac{\left(h_{r}+c_{r} k_{r}\right) K Q_{0}-\sqrt{\left(h_{r}+c_{r} k_{r}\right)^{2} K^{2} Q_{0}^{2}-2 h_{r} A_{r} D(K-1)^{2}}}{h_{r} D} .
$$

It can be seen from (17) that, if $K=1$, then $t^{*}(K)=0$. (12) is reduced to (6), if $K=1$, so (13) holds. The proof of Theorem 2 is complete.

Theorem 2 indicates that the supplier's cost as well as that of the system can be reduced in the proposed delay in payment policy, and the retailer's cost is not changed. In practice, the supplier will usually share the saving cost with the retailer, and the sharing rate is determined by their power of balance between them.

Theorem 3. The retailer's order size with coordination is greater than that without coordination; that is, $K \geq 1$.

Proof. Since

$$
\begin{aligned}
\frac{d^{2} t^{*}(K)}{d K^{2}}= & 2\left[\left(h_{r}+c_{r} k_{r}\right)^{2} K^{2} Q_{0}^{2}-2 h_{r} A_{r} D(K-1)^{2}\right]^{-3 / 2} \\
& \times\left(h_{r}+c_{r} k_{r}\right)^{2} h_{r} A_{r} D Q_{0}^{2}>0,
\end{aligned}
$$

$t^{*}(K)$ is convex in $K$. In addition, it is easy to verify that $d t^{*}(K) /\left.d K\right|_{K=1}=0$; so $t^{*}(K)$ is minimized, when $K=1$. In practice, the more the retailer orders, the longer the delay period should be; so $t^{*}(K)$ should increase in $K$. It is straight forward that only if $K \geq 1, t^{*}(K)$ is increasing in $K$. As a result, $K \geq 1$ holds. The proof of Theorem 3 is complete.

Theorem 3 demonstrates that delay in payments policy can indeed induce the retailer to increase his/her order quantity.

Then, we will prove $t^{*}(K) \leq K t_{0}$.

Proposition 4. If $s_{r}>2 c_{r} k_{r}$, then $0 \leq t^{*}(K) \leq K t_{0}$ holds for $1 \leq K \leq K_{1}$, where $K_{1}=1 /\left(1-\sqrt{\left(h_{r}+2 c_{r} k_{r}\right) /\left(h_{r}+s_{r}\right)}\right)$.

Proof. The condition for the existence of $t^{*}(K)$ is

$$
\left(h_{r}+c_{r} k_{r}\right)^{2} Q_{0}^{2} K^{2}-2 h_{r} A_{r} D(K-1)^{2} \geq 0
$$

that is

$$
\frac{\left(h_{r}+c_{r} k_{r}\right)^{2}}{h_{r}\left(h_{r}+s_{r}\right)} \geq \frac{(K-1)^{2}}{K^{2}} .
$$

In addition, to prove that $t^{*}(K) \leq K Q_{0} / D$ holds, that is,

$$
\begin{aligned}
& \frac{\left(h_{r}+c_{r} k_{r}\right) K Q_{0}-\sqrt{\left(h_{r}+c_{r} k_{r}\right)^{2} K^{2} Q_{0}^{2}-2 h_{r} A_{r} D(K-1)^{2}}}{h_{r} D} \\
& \quad \leq \frac{K Q_{0}}{D}
\end{aligned}
$$

we only need to prove that the following inequality holds:

$$
\frac{h_{r}+2 c_{r} k_{r}}{h_{r}+s_{r}} \geq \frac{(K-1)^{2}}{K^{2}} .
$$

By $\left(h_{r}+c_{r} k_{r}\right)^{2} / h_{r}\left(h_{r}+s_{r}\right) \geq\left(h_{r}+2 c_{r} k_{r}\right) /\left(h_{r}+s_{r}\right)$, we know whether $\left(h_{r}+2 c_{r} k_{r}\right) /\left(h_{r}+s_{r}\right) \geq(K-1)^{2} / K^{2}$; then $\left(h_{r}+c_{r} k_{r}\right)^{2} / h_{r}\left(h_{r}+s_{r}\right) \geq(K-1)^{2} / K^{2}$ holds. Therefore,

$$
1 \leq K \leq \frac{1}{1-\sqrt{\left(h_{r}+2 c_{r} k_{r}\right) /\left(h_{r}+s_{r}\right)}} .
$$

Set $K_{1}=1 /\left(1-\sqrt{\left(h_{r}+2 c_{r} k_{r}\right) /\left(h_{r}+s_{r}\right)}\right)$. By $K \geq 1$, we have $K_{1} \geq 1$; that is, $s_{r}>2 c_{r} k_{r}$. If $s_{r}>2 c_{r} k_{r}$ and (23) holds, it is easy to verify that $t^{*}(K)$ exists and $t^{*}(K) \leq K t_{0}$. The proof of Proposition 4 is complete.

Proposition 4 shows that when the retailer's storage cost is large, to reduce the storage cost, the retailer will not order too much items in the beginning of every ordering cycle. Accordingly, the trade credit period offered by the supplier will be less than the ordering cycle of the retailer.

Next, we will determine the supplier and the retailer's optimal ordering quantities $K^{*}$ and $\lambda^{*}$.

By substituting (17) into (12), we have

$\min \overline{T C_{s}}(K, \lambda)=\frac{A_{s} D}{\lambda K Q_{0}}+\left[\frac{\left(h_{s}+s_{s}\right)(\lambda-1)}{2}\right.$

$$
\left.+\frac{\left(c_{r} k_{s}+h_{s}\right)\left(h_{r}+c_{r} k_{r}\right)}{h_{r}}\right] K Q_{0}
$$

$$
\begin{aligned}
-\frac{\left(c_{r} k_{s}+h_{s}\right)}{h_{r}} \times( & \left(h_{r}+c_{r} k_{r}\right)^{2} K^{2} Q_{0}^{2} \\
& \left.-2 h_{r} A_{r} D(K-1)^{2}\right)^{1 / 2}
\end{aligned}
$$

s.t. $\quad \lambda K \frac{Q_{0}}{D} \leq L, \quad \lambda \geq 1$.

Since

$$
\begin{aligned}
& \frac{\partial^{2} \overline{T C_{s}}(K, \lambda)}{\partial K^{2}} \\
& =\frac{2 A_{s} D}{\lambda Q_{0} K^{3}}+2\left(c_{r} k_{s}+h_{s}\right)\left(h_{r}+c_{r} k_{r}\right)^{2} A_{r} D Q_{0}^{2} \\
& \quad \times\left[\left(h_{r}+c_{r} k_{r}\right)^{2} Q_{0}^{2} K^{2}-2 h_{r} A_{r} D(K-1)^{2}\right]^{-3 / 2}>0,
\end{aligned}
$$


then $\overline{T C_{s}}(K, \lambda)$ is convex in $K$, for $1 \leq K \leq K_{1}$. Let $K_{1}^{*}$ and $\lambda_{1}^{*}$ be the solutions of $\min \overline{T C_{s}}(K, \lambda)$, then, for given $\lambda, K_{1}^{*}(\lambda)$ satisfies $\partial \overline{T C_{s}}(K, \lambda) / \partial K=0$.

Since $\lambda$ is an integer and $\lambda \leq L / t_{0}$, we can get $K_{1}^{*}$ and $\lambda_{1}^{*}$ by the enumeration method.

Therefore, we have the following results:

(a) if $1 \leq K_{1}^{*}<K_{1}$, then $K^{*}=K_{1}^{*}$ and $\lambda^{*}=\lambda_{1}^{*}$;

(b) if $K_{1}^{*} \geq K_{1}$, then $K^{*}=K_{1}$.

If $K_{1}^{*} \geq K_{1}$, then $K^{*}=K_{1}$. By substituting $K^{*}=K_{1}$ into (24), we get

$\min \overline{T C_{s}}(\lambda)$

$$
\begin{aligned}
= & \frac{A_{s} D}{\lambda Q_{0}}\left(1-\sqrt{\frac{h_{r}+2 c_{r} k_{r}}{h_{r}+s_{r}}}\right) \\
& +\left[\frac{h_{s}+s_{s}}{2}(\lambda-1)+\frac{\left[c_{r} k_{s}+h_{s}\right]\left(h_{r}+c_{r} k_{r}\right)}{h_{r}}\right] \\
& \times \frac{Q_{0}}{1-\sqrt{\left(h_{r}+2 c_{r} k_{r}\right) /\left(h_{r}+s_{r}\right)}} \\
& -\frac{c_{r} k_{s}+h_{s}}{h_{r}}\left(\left[\frac{\left(h_{r}+c_{r} k_{r}\right) Q_{0}}{1-\sqrt{\left(h_{r}+2 c_{r} k_{r}\right) /\left(h_{r}+s_{r}\right)}}\right]^{2}\right. \\
\text { s.t. } \left.\quad \frac{2 h_{r} A_{r} D\left(h_{r}+2 c_{r} k_{r}\right)}{\left(\sqrt{h_{r}+s_{r}}-\sqrt{\left.h_{r}+2 c_{r} k_{r}\right)^{2}}\right.}\right)^{1 / 2} & \lambda \quad \frac{Q_{0}}{D} \leq L, \quad \lambda \geq 1 .
\end{aligned}
$$

It is easy to verify that $\overline{T C_{s}}(\lambda)$ is convex in $\lambda$. Similar to the Proof of Lemma1, we can get that if $L \geq$ $\sqrt{2 A_{r}} / \sqrt{D}\left(\sqrt{h_{r}+s_{r}}-\sqrt{h_{r}+2 c_{r} k_{r}}\right), \lambda^{*}=\min \left\{\lambda_{2}^{*}, \lambda_{3}^{*}\right\}$, where

$$
\begin{aligned}
& \lambda_{2}^{*}=\left[\sqrt{\frac{1}{4}+\frac{A_{s}}{A_{r}}\left(1-\sqrt{\frac{h_{r}+2 c_{r} k_{r}}{h_{r}+s_{r}}}\right)^{2}}-\frac{1}{2}\right], \\
& \lambda_{3}^{*}=\left[\frac{L \sqrt{D}\left(\sqrt{h_{r}+s_{r}}-\sqrt{h_{r}+2 c_{r} k_{r}}\right)}{\sqrt{2 A_{r}}}\right],
\end{aligned}
$$

$\lceil x\rceil$ is the least integer greater than or equal to $x ;[x]$ denotes the integer part of $x$.
Case II. When $t(K) \geq K t_{0}, H_{r}(K)=0$.

In this case, (11) is reduced to the following problem:

$$
\begin{array}{ll}
\min \quad & \overline{T C_{s}}(K, \lambda)=\frac{D A_{s}}{\lambda K Q_{0}}+\frac{\left(h_{s}+s_{s}\right) K Q_{0}(\lambda-1)}{2} \\
& +h_{s} D t(K)+c_{r} k_{s} D t(K) \\
\text { s.t. } \quad & \lambda K \frac{Q_{0}}{D} \leq L \\
& \frac{D A_{r}}{K Q_{0}}+\frac{s_{r} K Q_{0}}{2}-c_{r} k_{r} D t(K) \leq \sqrt{2 D A_{r}\left(h_{r}+s_{r}\right)}
\end{array}
$$$$
\lambda \geq 1
$$

By the second constraint of (28), we have

$$
t(K) \geq \frac{A_{r}}{K Q_{0} c_{r} k_{r}}+\frac{s_{r} K Q_{0}}{2 D c_{r} k_{r}}-\frac{Q_{0}\left(h_{r}+s_{r}\right)}{D c_{r} k_{r}} .
$$

Set $t_{3}=A_{r} / K Q_{0} c_{r} k_{r}+s_{r} K Q_{0} / 2 D c_{r} k_{r}-Q_{0}\left(h_{r}+s_{r}\right) / D c_{r} k_{r}$. In this case, $t(K) \geq K t_{0}$ holds. So,

$$
t(K) \geq \max \left\{K t_{0}, t_{3}\right\} .
$$

Let $F(K)=t_{3}-K t_{0}$; that is

$$
F(K)=\frac{A_{r}}{K Q_{0} c_{r} k_{r}}+\frac{s_{r} t_{0} K}{2 c_{r} k_{r}}-\frac{t_{0}\left(h_{r}+s_{r}\right)}{c_{r} k_{r}}-K \frac{Q_{0}}{D} .
$$

It is easy to verify that $F(K)$ is convex in $K$ and $F(K=1)<0$. Let $K_{2}$ and $K_{3}\left(K_{2}>K_{3}\right)$ be the solutions of $F(K)=0$; then

$$
\begin{gathered}
K_{2}=\frac{\sqrt{2 D A_{r}}}{1-\sqrt{\left(h_{r}+2 c_{r} k_{r}\right) /\left(h_{r}+s_{r}\right)}}>1, \\
K_{3}=\frac{\sqrt{2 D A_{r}}}{1+\sqrt{\left(h_{r}+2 c_{r} k_{r}\right) /\left(h_{r}+s_{r}\right)}}<1 .
\end{gathered}
$$

Therefore, if $1 \leq K \leq K_{2}$, then $t_{3} \leq K t_{0}, t^{*}(K)=K t_{0}$; if $K>K_{2}$, then $t_{3}>K t_{0}, t^{*}(K)=t_{3}$.

The following analysis is conducted based on the following two situations.

$$
\text { (i) If } 1 \leq K \leq K_{2}, t^{*}(K)=K t_{0} .
$$

It is straight forward that, in this case, the retailer's cost will reduce no matter how much the parameters change. By substituting $t^{*}(K)$ into (28), we get the following problem:

$$
\begin{array}{ll}
\min & \overline{T C_{s}}(K, \lambda)=\frac{D A_{s}}{\lambda K Q_{0}}+\left[\frac{h_{s}+s_{s}}{2}(\lambda-1)+h_{s}+c_{r} k_{s}\right] K Q_{0} \\
\text { s.t. } & \lambda K \frac{Q_{0}}{D} \leq L, \quad \lambda \geq 1 .
\end{array}
$$


Theorem 5. If $h_{s}+c_{r} k_{s}-\left(h_{s}+s_{s}\right) / 2<0$ and

$$
K^{*} \geq-\frac{h_{s}+s_{s}}{h_{s}-s_{s}+2 c_{r} k_{s}},
$$

then

$$
\overline{T C_{s}}\left(K^{*}, \lambda^{*}\right) \leq T C_{s}\left(\mu^{*}\right) .
$$

Proof. Let $\rho=\lambda K$; (33) is equivalent to the following problem:

$$
\begin{aligned}
\min \quad \overline{T C_{s}}(\rho)= & \frac{D A_{s}}{\rho Q_{0}}+\frac{h_{s}+s_{s}}{2}(\rho-1) Q_{0} \\
& +\left[h_{s}+c_{r} k_{s}-\frac{h_{s}+s_{s}}{2}\right] K Q_{0}+\frac{h_{s}+s_{s}}{2} Q_{0}
\end{aligned}
$$

s.t. $\quad \rho t_{0} \leq L, \quad \rho \geq 1$.

It is easy to verify that if $h_{s}+c_{r} k_{s}-\left(h_{s}+s_{s}\right) / 2<0$ and $K^{*} \geq$ $-\left(h_{s}+s_{s}\right) /\left(h_{s}-s_{s}+2 c_{r} k_{s}\right)$, then $\overline{T C_{s}}\left(K^{*}, \lambda^{*}\right) \leq T C_{s}\left(\mu^{*}\right)$. The proof of Theorem 5 is complete.

Theorem 5 indicates that when the retailer is required to settle the balance after the ordering cycle, the retailer's average cost will be reduced, but the supplier will benefit from coordination only under certain conditions.

Next, we will determine the supplier and the retailer's optimal ordering quantities.

It is easy to verify that $\overline{T C_{s}}(K, \lambda)$ is convex in $K$ for $1 \leq$ $K \leq K_{2}$. Let $K_{2}^{*}$ be the solution of $\partial \overline{T C_{s}}(K, \lambda) / \partial K=0$.

(a) If $K_{2}^{*} \geq K_{2}$, then $K^{*}=K_{2}$.

By substituting $K^{*}=K_{2}$ into (33), we have

$$
\begin{aligned}
& \min \overline{T C_{s}}(\lambda)= \frac{D A_{s}\left[1-\sqrt{\left(h_{r}+2 c_{r} k_{r}\right) /\left(h_{r}+s_{r}\right)}\right]}{\lambda Q_{0} \sqrt{2 D A_{r}}} \\
&+\left[\frac{h_{s}+s_{s}}{2}(\lambda-1)+h_{s}+c_{r} k_{s}\right] \\
& \times \frac{Q_{0} \sqrt{2 D A_{r}}}{1-\sqrt{\left(h_{r}+2 c_{r} k_{r}\right) /\left(h_{r}+s_{r}\right)}} \\
& \text { s.t. } \quad \frac{\lambda \sqrt{2 D A_{r}}}{1-\sqrt{\left(h_{r}+2 c_{r} k_{r}\right) /\left(h_{r}+s_{r}\right)}} \frac{Q_{0}}{D} \leq L, \quad \lambda \geq 1 .
\end{aligned}
$$

We can easily verify that $\overline{T C_{s}}(\lambda)$ is convex in $\lambda$. Similar to the Proof of Lemma 1, we can get the following result: if $L \geq$ $2 A_{r} /\left(\sqrt{h_{r}+s_{r}}-\sqrt{h_{r}+2 c_{r} k_{r}}\right), \lambda^{*}=\min \left\{\lambda_{4}^{*}, \lambda_{5}^{*}\right\}$, where

$$
\begin{aligned}
& \lambda_{4}^{*}=\left[\sqrt{\frac{1}{4}+\frac{A_{s}}{2 D A_{r}^{2}}\left(1-\sqrt{\frac{h_{r}+2 c_{r} k_{r}}{h_{r}+s_{r}}}\right)^{2}}-\frac{1}{2}\right], \\
& \lambda_{5}^{*}=\left[\frac{L\left(\sqrt{h_{r}+s_{r}}-\sqrt{h_{r}+2 c_{r} k_{r}}\right)}{2 A_{r}}\right],
\end{aligned}
$$

$\lceil x\rceil$ is the least integer greater than or equal to $x ;[x]$ denotes the integer part of $x$.

(b) If $1 \leq K_{2}^{*}<K_{2}$, then $K^{*}=K_{2}^{*}$.

By $\partial \overline{T_{s}}(K, \lambda) / \partial K=0$, we have

$$
K_{2}^{*}=\sqrt{\frac{\left(h_{r}+s_{r}\right) A_{s}}{2 A_{r} \lambda\left[\left(\left(h_{s}+s_{s}\right) / 2\right)(\lambda-1)+h_{s}+c_{r} k_{s}\right]}} .
$$

Substituting (39) into (33), we have

$$
\begin{array}{ll}
\min & \overline{T C_{s}}(\lambda)=2 \sqrt{\frac{A_{s} D\left[\left(\left(h_{s}+s_{s}\right) / 2\right)(\lambda-1)+h_{s}+c_{r} k_{s}\right]}{\lambda}} \\
\text { s.t. } & \frac{A_{s} \lambda}{D\left[\left(\left(h_{s}+s_{s}\right) / 2\right)(\lambda-1)+h_{s}+c_{r} k_{s}\right]} \leq L^{2}, \quad \lambda \geq 1 .
\end{array}
$$

Since $\sqrt{x}$ is strictly increasing in $x$, so (40) is equivalent to the following problem:

$$
\begin{array}{ll}
\min & \widetilde{T C_{s}}(\lambda)=\frac{A_{s} D\left[\left(\left(h_{s}+s_{s}\right) / 2\right)(\lambda-1)+h_{s}+c_{r} k_{s}\right]}{\lambda} \\
\text { s.t. } & {\left[\frac{L^{2} D\left(h_{s}+s_{s}\right)}{2}-A_{s}\right] \lambda+L^{2} D\left[h_{s}+c_{r} k_{s}-\frac{h_{s}+s_{s}}{2}\right] \geq 0}
\end{array}
$$$$
\lambda \geq 1 \text {. }
$$

Since $h_{s}+c_{r} k_{s}-\left(h_{s}+s_{s}\right) / 2<0$, then $\widetilde{T C_{s}}(\lambda)$ is increasing in $\lambda$. If $h_{s}+c_{r} k_{s}<\left(h_{s}+s_{s}\right) / 2$ and $L^{2} D\left(h_{s}+s_{s}\right) / 2-A_{s}>0$, then the first constraint of (41) holds for $\lambda \geq\left\lceil-\left[h_{s}+c_{r} k_{s}-\left(h_{s}+s_{s}\right) / 2\right] /\left(\left(h_{s}+\right.\right.\right.$ $\left.\left.\left.s_{s}\right) / 2-A_{s} / L^{2} D\right)\right\rceil$. Due to the fact that $T C_{s}(\lambda)$ is increasing in $\lambda$ and $\left\lceil-\left[h_{s}+c_{r} k_{s}-\left(h_{s}+s_{s}\right) / 2\right] /\left(\left(h_{s}+s_{s}\right) / 2-A_{s} / L^{2} D\right)\right\rceil \geq 1$, it is straight forward that $\lambda^{*}=\left\lceil-\left[h_{s}+c_{r} k_{s}-\left(h_{s}+s_{s}\right) / 2\right] /\left(\left(h_{s}+\right.\right.\right.$ $\left.\left.\left.s_{s}\right) / 2-A_{s} / L^{2} D\right)\right\rceil$.

$$
\text { (ii) If } K>K_{2} \text {, }
$$

$$
t^{*}(K)=t_{3}=\frac{A_{r}}{K Q_{0} c_{r} k_{r}}+\frac{s_{r} t_{0} K}{2 c_{r} k_{r}}-\frac{t_{0}\left(h_{r}+s_{r}\right)}{c_{r} k_{r}} .
$$

By substituting (42) into (28), the problem is reduced to

$$
\begin{aligned}
\min \overline{T_{s}}(K, \lambda)= & {\left[\frac{h_{s}+s_{s}}{2}(\lambda-1)+\frac{\left(h_{s}+c_{r} k_{s}\right) s_{r}}{2 c_{r} k_{r}}\right] K Q_{0} } \\
& +\left[\frac{A_{s} D}{\lambda}+\frac{\left(h_{s}+c_{r} k_{s}\right) A_{r} D}{c_{r} k_{r}}\right] \frac{1}{K Q_{0}} \\
& -\frac{\left(h_{s}+c_{r} k_{s}\right) \sqrt{2 D A_{r}\left(h_{r}+s_{r}\right)}}{c_{r} k_{r}}
\end{aligned}
$$

s.t. $\quad \lambda K \frac{Q_{0}}{D} \leq L, \quad \lambda \geq 1$. 
TABLE 1: Sample computing results for different values of $A_{s}$.

\begin{tabular}{lcccccccccc}
\hline$A_{s}$ & $\mu^{*}$ & $\mathrm{TC}_{s}\left(\mu^{*}\right)$ & $K^{*}$ & $\lambda^{*}$ & $t^{*}($ day $)$ & $\overline{T C_{s}}\left(\lambda^{*}\right)$ & $\operatorname{SSP}_{0}(\%)$ & $\operatorname{RSP}_{0.5}(\%)$ & $\operatorname{SSP}_{0.5}(\%)$ & $\mathrm{TSP}(\%)$ \\
\hline 30 & 1 & 6037.38 & 1.43 & 1 & 0.35 & 4942.59 & 18.13 & 2.72 & 9.07 & 4.18 \\
50 & 1 & 10062.31 & 1.62 & 1 & 0.66 & 7582.70 & 24.64 & 6.16 & 12.32 & 8.21 \\
70 & 2 & 13006.46 & 1.77 & 1 & 0.98 & 9955.52 & 23.46 & 7.58 & 11.73 \\
90 & 2 & 15018.92 & 1.90 & 1 & 1.28 & 12145.70 & 19.13 & 7.14 & 9.57 & 8.18 \\
120 & 2 & 18037.62 & 2.06 & 1 & 1.70 & 15187.63 & 15.80 & 7.08 & 7.90 & 7.47 \\
150 & 2 & 21056.31 & 2.20 & 1 & 2.09 & 18016.92 & 14.43 & 7.55 & 7.22 \\
\hline
\end{tabular}

TABLE 2: Sample computing results for different values of $h_{s}$.

\begin{tabular}{lcccccccccc}
\hline$h_{s}$ & $\mu^{*}$ & $T C_{s}\left(\mu^{*}\right)$ & $K^{*}$ & $\lambda^{*}$ & $t^{*}($ day $)$ & $\overline{T C_{s}}\left(\lambda^{*}\right)$ & $\operatorname{SSP}_{0}(\%)$ & $\operatorname{RSP}_{0.5}(\%)$ & $\operatorname{SSP}_{0.5}(\%)$ & $\mathrm{TSP}(\%)$ \\
\hline 1 & 2 & 11888.43 & 2.30 & 1 & 2.40 & 8101.07 & 31.86 & 9.41 & 15.93 \\
2 & 2 & 12261.11 & 2.05 & 1 & 1.65 & 8916.85 & 27.28 & 8.31 & 13.64 \\
3 & 2 & 12633.78 & 1.89 & 1 & 1.24 & 9504.06 & 24.77 & 7.78 & 12.39 \\
4 & 2 & 13006.46 & 1.77 & 1 & 0.98 & 9955.52 & 23.46 & 7.58 & 11.73 \\
10 & 1 & 14087.23 & 1.45 & 1 & 0.38 & 11433.89 & 18.84 & 6.59 & 9.33 \\
15 & 1 & 14087.23 & 1.33 & 1 & 0.22 & 12027.18 & 14.62 & 5.12 & 7.42 & 7.31 \\
20 & 1 & 14087.23 & 1.27 & 1 & 0.15 & 12400.63 & 11.97 & 4.19 & 5.99 & 4.02 \\
25 & 1 & 14087.23 & 1.22 & 1 & 0.11 & 12658.55 & 10.14 & 3.55 & 5.07 & 4.18 \\
30 & 1 & 14087.23 & 1.19 & 1 & 0.08 & 12847.74 & 8.80 & 3.08 & 4.40 \\
\hline
\end{tabular}

According to the constraints in (43), we get $1 \leq \lambda \leq L D / K Q_{0}$. In order to guarantee that there exits the feasible solution for the problem (43), the lifetime needs to satisfy $L \geq K_{2}\left(Q_{0} / D\right)$; that is

$$
L \geq \frac{2 D A_{r}}{\sqrt{h_{r}+s_{r}}-\sqrt{h_{r}+2 c_{r} k_{r}}} .
$$

It is easy to verify that the right side of (44) is usually very large, so (44) is not easy to be satisfied for fixed lifetime products. Hence, for fixed lifetime products, providing longer credit period than the retailer's order period is not commonly applicable.

Remark 6. By (12) and (28), it is straightforward that for items with infinite lifetime, it is possible that $\overline{T C_{s}}(K, \lambda)$ is minimized when both $t(K)$ and $K$ are very large, which is meaningless in practice. So, an upper bound $\bar{t}$ is necessary to be set on delay period $t(K)$ to avoid very large delay period. But for items with finite lifetime, the first constraints of (12) and (28) ensure that $K$ will not be very large. Particularly, in Case I $\left(0 \leq t(K) \leq K t_{0}\right)$, by $(17), t^{*}(K)$ will not be very large. In Case II $\left(t(K) \geq K t_{0}\right)$, we have $t^{*}(K)=K t_{0}$, for $1 \leq K \leq$ $K_{2}$. And we have proved that $K>K_{2}$ is not applicable for fixed lifetime products. Hence, the upper bound on $t$ is not necessary any more for fixed lifetime products.

\section{Numerical Example}

In this section, numerical examples are presented to evaluate the benefit of the proposed policy in Section 2. Moreover, we will examine the implications of the change in the value of parameters.

Let $L=0.2$ year and $D=150000$ units per year. $\operatorname{SSP}_{\alpha}(\%)$ and $\operatorname{RSP}_{\alpha}(\%)$ denote the savings in percentage of the supplier and the retailer when the supplier shares $\alpha$ percentage of his/her cost saving with the retailer and let $\alpha=0.5$. TSP(\%) denotes the saving in percentage of the supply chain.

Case I (example). In Case I, we prove that the retailer's cost with coordination is the same as that without coordination, but the supplier's cost is reduced. If the supplier would like to share the saving cost $\left(T C_{s}-\overline{T C_{s}}\right)$ with the retailer, the retailer's cost can also be reduced.

Let $A_{s}=\$ 70$ per order, $A_{r}=\$ 50$ per order, $c_{r}=\$ 10$ per unit, $h_{s}=\$ 4$ per unit per year, $h_{r}=\$ 7$ per unit per year, $s_{s}=\$ 12$ per unit per year, $s_{r}=\$ 20$ per unit per year, $k_{s}=\$ 0.1$ per dollar per year, and $k_{r}=\$ 0.1$ per dollar per year.

When the values of $A_{s}, h_{s}$, and $k_{s}$ change, the corresponding computing results are as specified in Tables $1-3$.

As illustrated in Table 1 , as $A_{s}$ increases, $K^{*}, t^{*}$, and the supplier's cost without and with coordination all increase. In fact, when the supplier's ordering cost $A_{s}$ is high, the supplier will reduce his/her ordering cost by increasing the order quantity per cycle, that is, increasing $\mu^{*}$ in the absence of coordination or $K^{*}$ in the presence of coordination. In order to entice the retailer to order more, the supplier will provide a longer credit period $t^{*}$. Moreover, as $A_{s}$ increases, the supplier and the retailer's savings in percentage decrease or increase, but they are both positive. The total cost saving of the supply chain is also positive; that is, the proposed policy is effective to the system.

By Table 2, as $h_{s}$ increases, $K^{*}$ and $t^{*}$ both decrease, but the supplier's cost without and with coordination both increase. Actually, if the supplier's capital cost is high, then he/she will provide a shorter delay period to reduce his/her capital cost. The shorter credit period offered by the supplier will cause the less order multiple of the retailer. In addition, as $h_{s}$ increases, the supplier and the retailer's savings in 
TABLE 3: Sample computing results for different values of $k_{s}$.

\begin{tabular}{lccccccccc}
\hline$k_{s}$ & $\mu^{*}$ & $K^{*}$ & $\lambda^{*}$ & $t^{*}($ day $)$ & $\overline{T C_{s}}\left(\lambda^{*}\right)$ & $\operatorname{SSP}_{0}(\%)$ & $\operatorname{RSP}_{0.5}(\%)$ & $\operatorname{SSP}_{0.5}(\%)$ & $\mathrm{TSP}^{*}(\%)$ \\
\hline 0.05 & 2 & 1.82 & 1 & 1.09 & 9743.23 & 25.09 & 8.11 & 7.54 & 9.85 \\
0.1 & 2 & 1.77 & 1 & 0.98 & 9955.52 & 23.46 & 7.58 & 11.73 \\
0.15 & 2 & 1.73 & 1 & 0.88 & 10145.69 & 22.00 & 7.11 & 11.00 \\
0.2 & 2 & 1.69 & 1 & 0.80 & 10317.34 & 20.68 & 6.68 & 10.34 \\
0.3 & 2 & 1.62 & 1 & 0.66 & 10615.78 & 18.38 & 5.94 & 8.63 \\
\hline
\end{tabular}

TABLE 4: Sample computing results for different values of $A_{r}$.

\begin{tabular}{lccccccccc}
\hline$A_{r}$ & $\lambda^{*}$ & $K^{*}$ & $K^{*} Q_{0}$ & $t^{*}($ day $)$ & $K^{*} Q_{0} / D($ day $)$ & $\mathrm{R}$ of $(34)$ & $\mathrm{RSP}_{0}(\%)$ & $\mathrm{SSP}_{0}(\%)$ & $\mathrm{TSP}(\%)$ \\
\hline 80 & 1 & 2.32048 & 2148.34 & 0.01432 & 0.01432 & 2.57143 & 12.15 & -1.68 & 5.03 \\
100 & 1 & 2.0755 & 2148.34 & 0.01432 & 0.01432 & 2.57143 & 16.61 & -17.31 \\
120 & 1 & 1.89466 & 2148.34 & 0.01432 & 0.01432 & 2.57143 & 19.48 & -19.18 \\
140 & 1 & 1.75412 & 2148.34 & 0.01432 & 0.01432 & 2.57143 & 21.38 & -20.02 & 3.06 \\
160 & 1 & 1.64083 & 2148.34 & 0.01432 & 0.01432 & 2.57143 & 22.65 & -20.17 \\
\hline
\end{tabular}

TABLE 5: Sample computing results for different values of $h_{r}$.

\begin{tabular}{cccccccccc}
\hline$h_{r}$ & $\lambda^{*}$ & $K^{*}$ & $K^{*} Q_{0}$ & $t^{*}($ day $)$ & $K^{*} Q_{0} / D($ day $)$ & $\mathrm{R}$ of $(34)$ & $\operatorname{RSP}_{0}(\%)$ & $\operatorname{SSP}_{0}(\%)$ & $\mathrm{TSP}(\%)$ \\
\hline 5 & 1 & 2.5 & 2449.49 & 0.01633 & 0.01633 & 1.6 & 5.26 & 5.36 & 5.19 \\
6 & 1 & 2.54951 & 2449.49 & 0.01633 & 0.01633 & 1.6 & 7.35 & 4.99 \\
7 & 1 & 2.598076 & 2449.49 & 0.01633 & 0.01633 & 1.6 & 9.39 & 4.66 \\
8 & 1 & 2.645751 & 2449.49 & 0.01633 & 0.01633 & 1.6 & 11.40 & 4.37 \\
9 & 1 & 2.692582 & 2449.49 & 0.01633 & 0.01633 & 1.6 & 13.37 & 4.12 \\
\hline
\end{tabular}

percentage both decrease. Hence, if the supplier's holding cost is high, the supplier and the retailer cannot gain much from coordination.

From Table 3, as $k_{s}$ increases, the supplier's cost with coordination increases, but $K^{*}, t^{*}$, and the supplier and the retailer's savings in percentage decrease. If $k_{s}$ is large, it is profitable for the supplier to invest, and accordingly the credit period offered to the retailer will be shortened. In this case, the supplier and the retailer cannot gain much from coordination.

Case II (example). In Case II, we prove that if $1 \leq K \leq K_{2}$, the retailer's cost with coordination is less than that without coordination no matter how much the parameters change, but the supplier will benefit only if (34) holds. In Tables 4 and 5 , "R of (34)" denotes the right hand side of (34).

(1) Let $A_{s}=\$ 200$ per order, $c_{r}=\$ 5$ per unit, $h_{s}=\$ 6$ per unit per year, $h_{r}=\$ 8$ per unit per year, $s_{s}=\$ 12$ per unit per year, $s_{r}=\$ 20$ per unit per year, $k_{s}=\$ 0.1$ per dollar per year, and $k_{r}=\$ 0.1$ per dollar per year. The different values of $A_{r}$ and the computing results are as specified in Table 4 .

As shown in Table 4, when $K^{*}$ is less than " $\mathrm{R}$ of (34)," the supplier's cost saving in percentage is negative, but the retailer's is positive. It is consistent with Theorem 5; that is, if (34) does not hold, the supplier will not benefit from coordination.

(2) Let $A_{s}=\$ 200$ per order, $A_{r}=\$ 80$ per order, $c_{r}=$ $\$ 10$ per unit, $s_{s}=\$ 12$ per unit per year, $s_{r}=\$ 20$ per unit per year, $h_{s}=\$ 4$ per unit per year, $k_{s}=\$ 0.1$ per dollar per year, and $k_{r}=\$ 0.1$ per dollar per year. The different values of $h_{r}$ and the computing results are as specified in Table 5 .

In Table $5, K^{*}$ is greater than " $\mathrm{R}$ of (34)." That is, (34) holds. In this case, we observe that the supplier and the retailer's cost savings in percentage are both positive and increase as $h_{r}$ increases. Hence, if the retailer's holding cost is high, both parties can gain much from coordination.

In Case II, the retailer's cost decreases, but the supplier's cost possibly decreases or increases, which depends on the value of the parameters and $K^{*}$. As proved in Theorem 5, if (34) holds, then the supplier's cost can be reduced by coordination as shown in Table 5, else as illustrated in Table 4, the supplier's average cost increases.

\section{Conclusions}

In this paper, supply chain coordination for fixed lifetime products through delay in payments is considered and modeled. The analytical method as to how to determine the optimal strategy is proposed, and analytically tractable solutions are obtained. It is indicated that if the retailer is required to settle the balance with the supplier before the end of the ordering cycle, then the supplier can gain with coordination. The supplier's total cost as well as that of the total system can be reduced no matter how much the parameters change. Thus, the supplier can not only compensate the retailer for his increased inventory cost by offering the retailer an order 
size dependent delay period, but also provide the retailer with an additional saving of $\alpha\left(T C_{s}\left(\mu^{*}\right)-\overline{T C_{s}}\left(K^{*}, \lambda^{*}\right)\right)$. Here, $\alpha$ is determined through negotiations between the supplier and the retailer and is generally dependent on the existing balance of power between them. Furthermore, it is indicated that, in this case, the retailer's order size is larger at cooperation against noncooperation $(K>1)$. As proved in Theorem 5 , if the retailer is permitted to settle the balance after the ordering cycle, the supplier can gain only under certain conditions. In this case, the credit period is equal to the ordering cycle.

In the proposed model, demand is assumed to be a deterministic constant. In fact, the demand is influenced by many factors, such as price [30], inventory level [31], advertising strategy [32], and other uncertainties or noises from market; so the varying or uncertain demand functions are more practical. However, the analysis of the models in these cases would be more complicated and we need to analyze the characteristics of noises first $[33,34]$. Perhaps it is difficult to obtain the analytical solutions, but one could try to obtain the optimal numerical solutions. Anyway, further works could extend the models in these ways.

\section{Conflict of Interests}

The authors declare that there is no conflict of interests regarding the publication of this paper.

\section{Acknowledgments}

The authors gratefully acknowledge the supports from the National Natural Science Foundation of China (71371139 and 71002020), the Shanghai Pujiang Program (12PJC069), the "Shu Guang" project supported by the Shanghai Municipal Education Commission and the Shanghai Education Development Foundation (13SG24), and the Fundamental Research Funds for the Central Universities.

\section{References}

[1] J. P. Monahan, "A quantity discount pricing model to increase vendor profit," Management Science, vol. 30, no. 6, pp. 720-726, 1984.

[2] H. L. Lee and M. J. Rosenblatt, "A generalized quantity discount pricing model to increase supplier's profits," Management Science, vol. 32, pp. 1177-1185, 1986.

[3] H. Shin and W. C. Benton, "Quantity discount-based inventory coordination: effectiveness and critical environmental factors," Production and Operations Management, vol. 13, no. 1, pp. 6376, 2004.

[4] M. Y. Jaber and I. H. Osman, "Coordinating a two-level supply chain with delay in payments and profit sharing," Computers and Industrial Engineering, vol. 50, no. 4, pp. 385-400, 2006.

[5] L.-H. Chen and F.-S. Kang, "Integrated vendor-buyer cooperative inventory models with variant permissible delay in payments," European Journal of Operational Research, vol. 183, no. 2, pp. 658-673, 2007.

[6] S. P. Sarmah, D. Acharya, and S. K. Goyal, "Coordination and profit sharing between a manufacturer and a buyer with target profit under credit option," European Journal of Operational Research, vol. 182, no. 3, pp. 1469-1478, 2007.

[7] H. Emmons and S. M. Gilbert, "The role of returns policies in pricing and inventory decisions for catalogue goods," Management Science, vol. 44, no. 2, pp. 276-283, 1998.

[8] I. Giannoccaro and P. Pontrandolfo, "Supply chain coordination by revenue sharing contracts," International Journal of Production Economics, vol. 89, no. 2, pp. 131-139, 2004.

[9] G. P. Cachon, "Supply chain coordination with contracts," in Handbooks in Operations Research and Management Science: Supply Chain Management, A. G. Kok and S. C. Graves, Eds., pp. 229-340, Elsevier, Amsterdam, The Netherlands, 2003.

[10] P. Kouvelis, C. Chambers, and H. Wang, "Supply chain management research and production and operations management: review, trends, and opportunities," Production and Operations Management, vol. 15, no. 3, pp. 449-469, 2006.

[11] S. Nahmias, "Perishable inventory theory: a review," Operations Research, vol. 30, no. 4, pp. 680-708, 1982.

[12] P. M. Ghare and G. H. Schrader, "A model for exponentially decaying inventory system," International Journal of Production Research, vol. 21, pp. 449-460, 1963.

[13] R. P. Covert and G. C. Philip, "An EOQ model for items with Weibull distribution deterioration," AIIE Transactions, vol. 5, no. 4, pp. 323-326, 1973.

[14] G. C. Philip, "A generalized EOQ model for items with Weibull distribution deterioration," AIIE Transactions, vol. 6, no. 2, pp. 159-162, 1974.

[15] A. K. Jalan, R. R. Giri, and K. S. Chaudhuri, "EOQ model for items with Weibull distribution deterioration, shortages and trended demand," International Journal of Systems Science, vol. 27, no. 9, pp. 851-855, 1996.

[16] P. L. Abad, "Optimal pricing and lot-sizing under conditions of perishability and partial backordering," Management Science, vol. 42, no. 8, pp. 1093-1104, 1996.

[17] P. L. Abad, "Optimal price and order size for a reseller under partial backordering," Computers and Operations Research, vol. 28, no. 1, pp. 53-65, 2000.

[18] S. K. Goyal and B. C. Giri, "Recent trends in modeling of deteriorating inventory," European Journal of Operational Research, vol. 134, no. 1, pp. 1-16, 2001.

[19] B. E. Fries, "Optimal ordering policy for a perishable commodity with fixed lifetime," Operations Research, vol. 23, no. 1, pp. 46-61, 1975.

[20] S. Nahmias, "Optimal ordering policies for perishable inventory," Operations Research, vol. 23, pp. 735-749, 1975.

[21] P. Nandarkumar and T. E. Morton, "Near myopic heuristic for the fixed life perishability problem," Management Science, vol. 39, pp. 1490-1498, 1993.

[22] L. Liu and Z. Lian, “ $(s, S)$ continuous review models for products with fixed lifetimes," Operations Research, vol. 47, no. 1, pp. 150$158,1999$.

[23] H. Hwang and K. H. Hahn, "An optimal procurement policy for items with an inventory level-dependent demand rate and fixed lifetime," European Journal of Operational Research, vol. 127, no. 3, pp. 537-545, 2000.

[24] Z. Lian and L. Liu, "Continuous review perishable inventory systems: models and heuristics," IIE Transactions, vol. 33, no. 9, pp. 809-822, 2001.

[25] E. Berk and Ü. Gürler, "Analysis of the (Q, r) inventory model for perishables with positive lead times and lost sales," Operations Research, vol. 56, no. 5, pp. 1238-1246, 2008. 
[26] F. Olsson and P. Tydesjö, "Inventory problems with perishable items: fixed lifetimes and backlogging," European Journal of Operational Research, vol. 202, no. 1, pp. 131-137, 2010.

[27] Y. Duan, J. Luo, and J. Huo, "Buyer-vendor inventory coordination with quantity discount incentive for fixed lifetime product," International Journal of Production Economics, vol. 128, no. 1, pp. 351-357, 2010.

[28] Y. Duan, J. Huo, Y. Zhang, and J. Zhang, "Two level supply chain coordination with delay in payments for fixed lifetime products," Computers \& Industrial Engineering, vol. 63, no. 2, pp. 456-463, 2012.

[29] H. Hwang and K. H. Hahn, "Optimal procurement policy for items with an inventory level-dependent demand rate and fixed lifetime," European Journal of Operational Research, vol. 127, no. 3, pp. 537-545, 2000.

[30] J.-T. Teng, C.-T. Chang, and S. K. Goyal, "Optimal pricing and ordering policy under permissible delay in payments," International Journal of Production Economics, vol. 97, no. 2, pp. 121-129, 2005.

[31] T. L. Urban, "Inventory models with inventory-level-dependent demand: a comprehensive review and unifying theory," European Journal of Operational Research, vol. 162, no. 3, pp. 792804, 2005.

[32] N. H. Shah, H. N. Soni, and K. A. Patel, "Optimizing inventory and marketing policy for non-instantaneous deteriorating items with generalized type deterioration and holding cost rates," Omega, vol. 41, pp. 421-430, 2013.

[33] M. Li and W. Zhao, "On bandlimitedness and lag-limitedness of fractional Gaussian noise," Physica A, vol. 392, no. 9, pp. 19551961, 2013.

[34] M. Li, "A class of negatively fractal dimensional gaussian random functions," Mathematical Problems in Engineering, vol. 2011, Article ID 291028, 18 pages, 2011. 


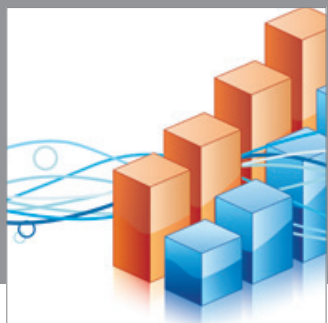

Advances in

Operations Research

mansans

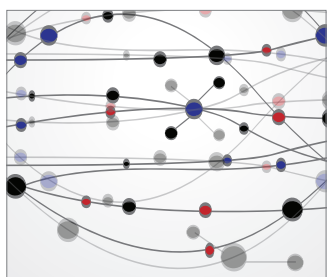

The Scientific World Journal
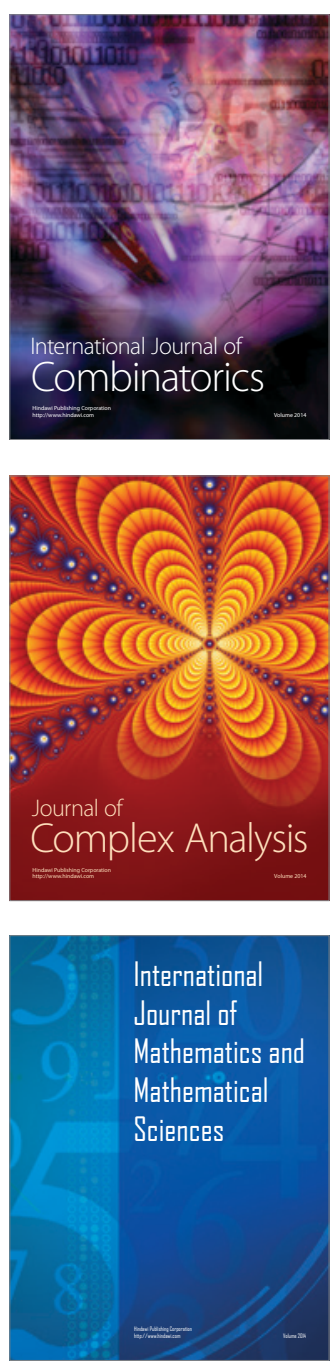
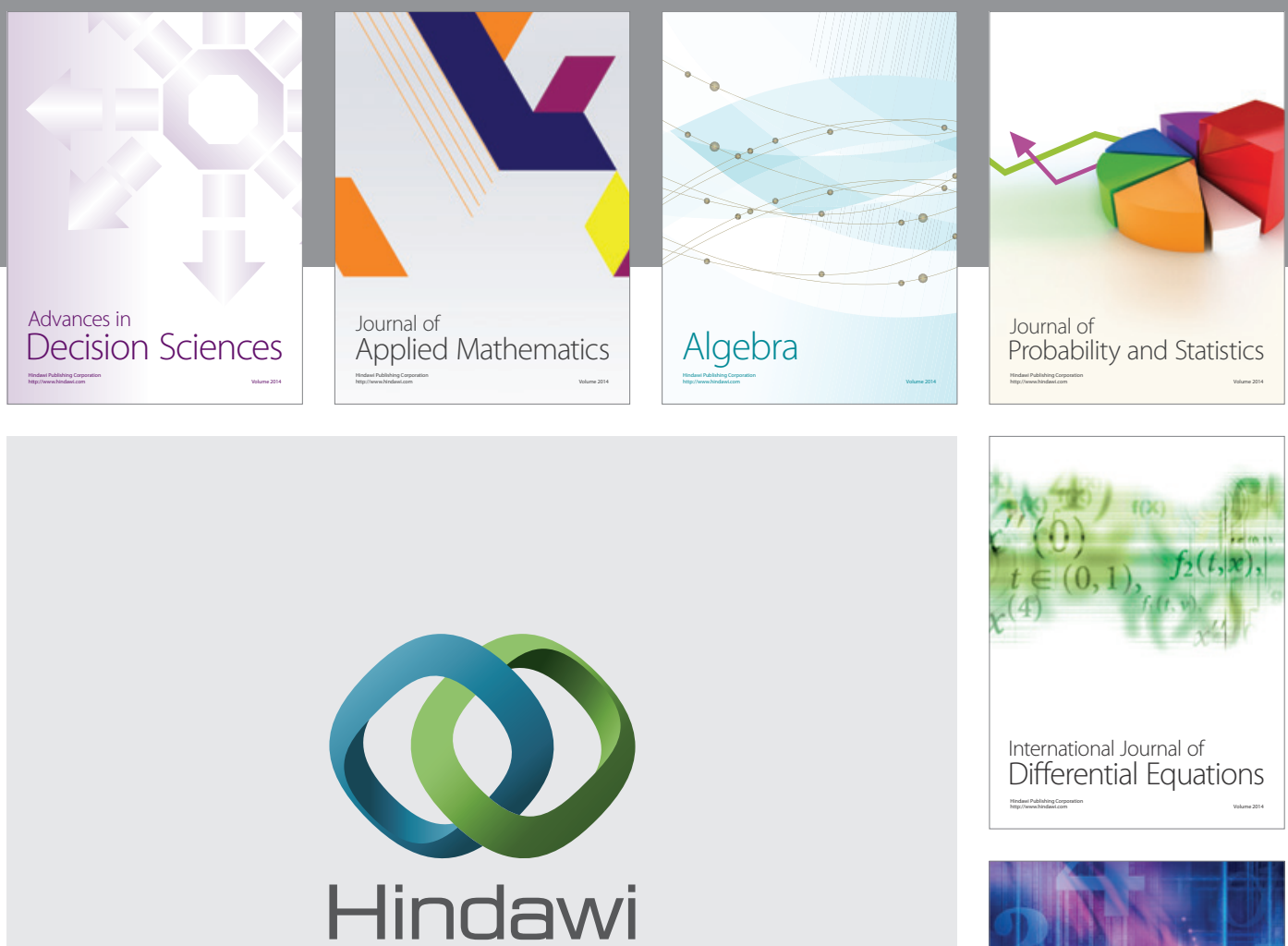

Submit your manuscripts at http://www.hindawi.com
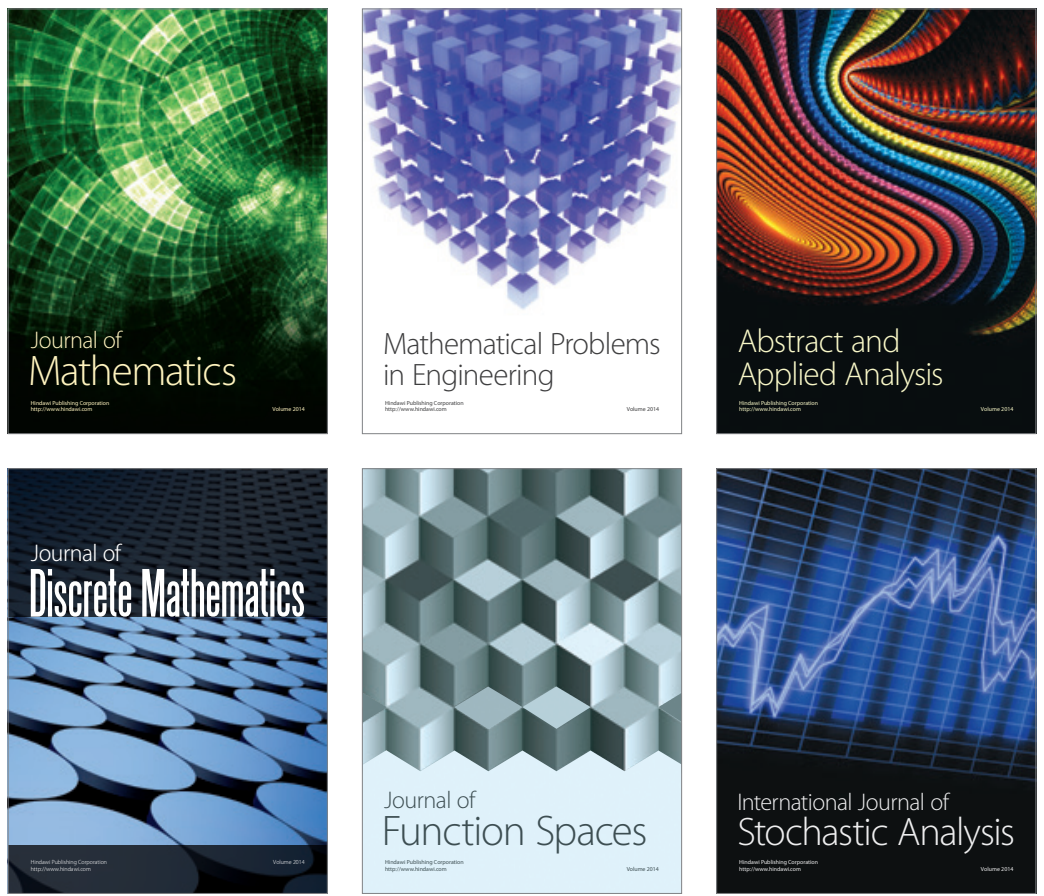

Journal of

Function Spaces

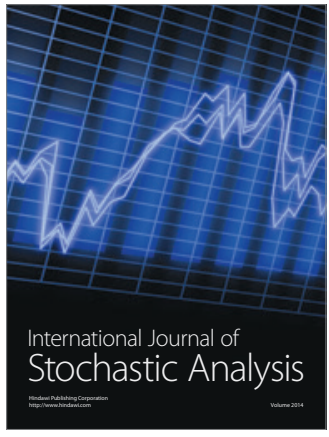

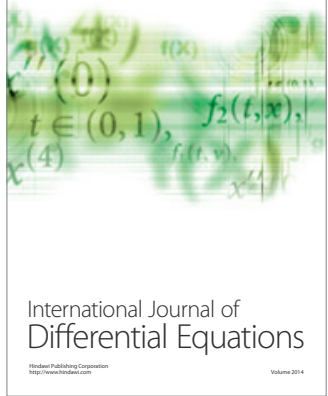
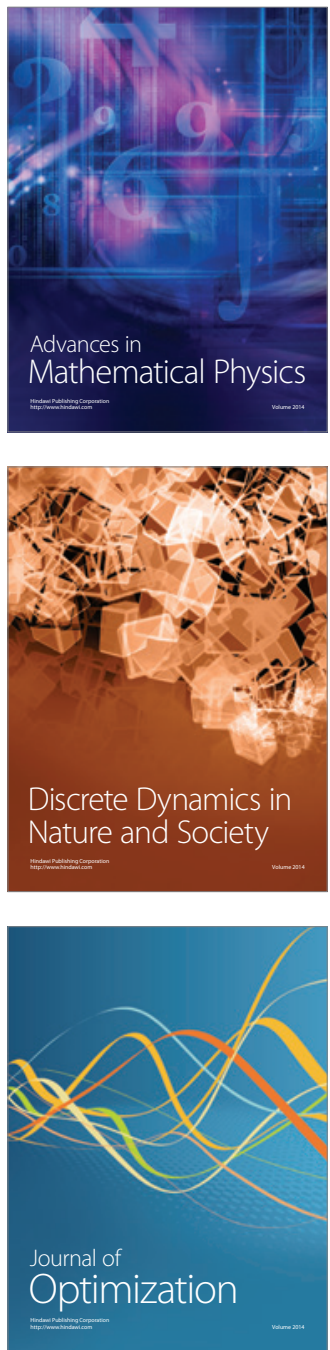